\title{
Measurement of the Temperature of Cold Highly Charged Ions Produced in an Electron Beam Ion Trap
}

\author{
P. Beiersdorfer \\ V. Decaux \\ K. Widmann \\ Prepared for Submittal to \\ 7th International Conference on the Physics of Highly Charged Ions \\ September 19-23, 1994, Vienna, Austria
}

September 14, 1994

This is a preprint of a paper intended for publication in a journal or proceedings. Since changes may be made before publication, this preprint is made available with the understanding that it will not be cited or reproduced without the permission of the author. 


\section{DISCLAIMER}

This document was prepared as an account of work sponsored by an agency of the United States Government. Neither the United States Government nor the Uni versity of California nor any of their employees, makes any warranty, express or implied, or assumes any legal liability or responsibility for the accuracy, completeness, or usefulness of any information, apparatus, product, or process disclosed, or represents that its use would not infringe privately owned rights. Reference herein to any specific commercial products, process, or service by trade name, trademark, manufacturer, or otherwise, does not necessarily constitute or imply its endorsement, recommendation, or favoring by the United States Government or the. University of California. The views and opinions of authors expressed herein do not necessarily state or reflect those of the United States Government or the University of California, and shall not be used for advertising or product endorsement purposes. 
7. International Conference on the Physics of Highly Charged Ions

September 19 - 23, 1994, Vienna, Austria

Paper: Mo62

\title{
Measurement of the Temperature of Cold Highly Charged Ions Produced in an Electron Beam Ion Trap
}

\author{
P. Beiersdorfer, V. Decaux, K. Widmann \\ Department of Physics and Space Technology \\ Lawrence Livermore National Laboratory, Livermore, CA 94551, USA
}

The temperature of highly charged titanium ions produced and trapped in an electron beam ion trap was determined by precisely measuring the broadening of the emission line profile caused by the thermal Doppler motion. The measured temperature ranges from about $700 \mathrm{eV}$ for deeply trapped ions to about $70 \mathrm{eV}$ for ions in a shallow trap. The latter value represents the lowest temperature at which the $\mathrm{x}$-ray emission of collisonally excited heliumlike $\mathrm{Ti}^{20+}$ ions has ever been recorded, and the measured transitions represent the narrowest $x$-ray lines observed from highly charged titanium ions.

Author to whom all correspondences should be sent:

Peter Beiersdorfer, Lawrence Livermore National Laboratory, P. O. Box 808, Mailstop L-421, Livermore, CA 94551, USA. Phone: (510) 423-3985, FAX (510) 422-5940. 


\section{Introduction}

Characteristic $\mathbf{x}$-ray lines of highly charged ions from virtually all plasma sources are broadened by the thermal motion of the emitting ions. In fact, the line broadening induced by the thermal motion represents a standard technique for measuring the ion temperature of hightemperature plasmas [1]. The line emission from laser-produced plasmas may be broadened further by opacity or density effects. The line emission observed in accelerator-based measurements is broadened by the Doppler effect, because the necessity for a finite acceptance angle entails viewing relativistic ions with different velocity components. The line emission from any of these sources may be broadened further by satellite lines populated by single or multiple electron capture into high-lying spectator levels. As a result of this broadening, the resolution and the precision with which measurements can be made is limited.

The electron beam ion trap (EBIT) facility uses a monoenergetic electron beam to produce and excite highly charged ions in a trap [2]. Because excitation processes can be selected by choosing the appropriate beam energies, the population of satellite transitions that may broaden a given line in plasma and accelerator sources are avoided [3]. Moreover, the electron density is less than $5 \times 10^{12} \mathrm{~cm}^{-3}$ so that opacity or density effects are unimportant and broadening effects that may affect high-density plasma sources are absent. On the other hand, interaction with the electron beam causes strong heating of the ions; thus ion thermal broadening is expected to play a role in determining the widths of $\mathrm{x}$-ray lines produced with an EBIT device [2]. The temperature of the ions, however, must be limited by the fact that ions with enough kinetic energy to overcome the trapping potential will leave the trap and are lost. By adjusting the trapping potential it should thus be possible to reduce the ion temperature. In the absence of any other line-broadening mechanism we thus expect to observe $\mathrm{x}$-ray lines with widths much less than achievable with any other source, provided the temperature of the ions trapped in the EBIT device is reduced sufficiently.

In the following, we present a measurement of the line widths of the transitions $\left(1 \mathrm{~s} 2 \mathrm{p}^{2}\right)_{5 / 2}$ $\rightarrow\left(1 s^{2} 2 p\right)_{3 / 2}$ and $\left(1 s 2 p^{2}\right)_{3 / 2} \rightarrow\left(1 s^{2} 2 p\right)_{1 / 2}$ in lithiumlike $\mathrm{Ti}^{19+}$. These transitions, in standard 


\section{DISCLAIMER}

Portions of this document may be illegible in electronic image products. Images are produced from the best available original document. 
notation dubbed $j$ and $k$, respectively, are excited by dielectronic recombination of a beam electron with heliumlike $\mathrm{Ti}^{20+}$ ions in the ground state, i.e., by the process

$$
1 s^{2}+e^{-} \rightarrow 1 s 2 p^{2} \rightarrow 1 s^{2} 2 p+h v .
$$

Because of the resonant nature of dielectronic recombination, this process occurs only at discrete electron energies, $3332 \mathrm{eV}$ for $j$ and $3329 \mathrm{eV}$ for $k$. There is a spread of about $50 \mathrm{eV}$ in the energy of the beam electrons [4] so that both transitions are excited simultaneously. The dielectronic resonance strengths of these lines are the strongest among the dielectronic resonances involving heliumlike $\mathrm{Ti}^{20+}$ ions [5]. Setting the electron beam energy to their resonance energy thus produces very intense lines that can be measured even when other transitions are too weak to be observed. In the following we show that by choosing the appropriate operating conditions the widths of these lines is reduced to $0.25 \mathrm{~m} \AA$ corresponding to an ion temperature of merely $70 \mathrm{eV}$. This is the lowest ion temperature for which these transitions have ever been observed, and the lines thus represent the narrowest $\mathrm{x}$-ray lines observed from highly charged titanium ions.

\section{Measurements}

The measurements were carried out with a von Hámos-type high-resolution crystal spectrometer [6]. A spectrometer based on this geometry functions like a flat-crystal spectrometer in the plane of dispersion but provides focusing for rays perpendicular to the plane of dispersion. To achieve this the analyzing crystal is bent cylindrically and placed such that the axis of the cylinder lies in the plane of dispersion. The von Hámos geometry requires a point or line $\mathrm{x}$-ray source. Because EBIT represents a slit-like line source, whose dimensions are determined by the 60- $\mu \mathrm{m}$ diameter electron beam and the 2-cm long trap length, such a spectrometer is ideally suited for high-resolution $\mathrm{x}$-ray measurements on this facility.

The resolving power of the spectrometer is determined by the radius of curvature of the crystal and thus by the overall dimensions of the spectrometer. In our measurements we employed 
a $120 \times 50 \times 0.25 \mathrm{~mm}^{3}$ quartz(203) crystal with a radius of curvature $R=240 \mathrm{~cm}$ and lattice spacing $2 d=2.749 \AA$. The nominal resolving power attainable with this spectrometer is $\lambda / \Delta \lambda=$ 22,000 at a Bragg angle of $\theta=45^{\circ}$, as shown in Ref. [7].

A spectrum of the two lithiumlike satellite transitions obtained under operating conditions standard for spectroscopy is shown in Fig. 1(a). Here, the beam current was $132 \mathrm{~mA}$. The applied trapping potential was $200 \mathrm{~V}$. The observed width of each line was $0.77 \mathrm{~m} \AA$, or $\lambda \Delta \lambda=$ 3400 , which was much wider than the limits given by the instrumental resolving power. Using the Doppler relation for line broadening

$$
T_{i}=m_{i} c^{2}[\Delta \lambda \lambda \lambda]^{2} / 8 \ln 2
$$

where $m_{i}$ is the ion mass and $c$ is the speed of light, we determined an ion temperature $T_{i}=685 \mathrm{eV}$ from the observed line width.

Departing from the usual operating parameters employed for spectroscopy we lowered the ion temperature by successively reducing and ultimately eliminating the axial trap. The geometry of the EBIT trap is such that ions may be trapped by the electric potential generated by the electron beam even in the absence of an applied axial potential [2]. At $\mathrm{I}=130 \mathrm{~mA}$ the effective trapping potential amounts to more than $40 \mathrm{~V}$. To counteract such trapping by the beam we lowered the beam current to $I=52 \mathrm{~mA}$ and set the axial potential to $V_{a x}=-20 \mathrm{~V}$. This reduced the measured line width to $0.25 \mathrm{~m} \AA$, as illustrated in Fig. 1(b). The value of $\lambda / \Delta \lambda$ achieved was 10,500 . The infered ion temperature was a mere $70 \mathrm{eV}$.

\section{Discussion}

Using similar instrumentation and procedures $\left(I=106 \mathrm{~mA}, \mathrm{~V}_{\mathrm{ax}}=-10 \mathrm{~V}\right)$, we had measured earlier an ion temperature of $130 \mathrm{eV}$ for heliumlike $\mathrm{Ti}^{20+}$ [7]. The present measurement lowers the temperature of the ions in EBIT by $40 \%$.

While lowering the beam current and the trapping potential is accompanied by a reduction of the intensity of the $x$-ray lines produced, the present measurements show that a very low ion 
temperature can be achieved while still providing adequate signal strength for spectroscopic measurements. In fact, the signal-to-background ratio in Fig. 1(b) was large enough that an additional loss in signal strength would not prevent further measurements. Moreover, the counting time was the same ( 11 hours) for each of the two spectra shown in Fig. 1.

The production of cold highly charged ions is not only of importance to high-resolution spectroscopy, but also of great importance to the success of experiments conducted with extracted

ions. For example, the spread of the ion velocities is a crucial parameter in capturing and thus in re-trapping of extracted ions [8], as well as for bunching of ions interacting in short bursts with surfaces [9]. Experiments are, therefore, planned to reduce the temperature of the ions trapped in EBIT further.

\section{Acknowledgments}

This work was performed under the auspices of the U.S. Department of Energy by the Lawrence Livermore National Laboratory under contract no. W-7405-ENG-48. 


\section{References}

[1] M. Bitter, S. von Goeler, R. Horton, M. Goldman, K. Hill, N. R. Sauthoff, and W. Stodiek, Phys. Rev. Lett. 42, 304 (1979).

[2] M. A. Levine, R. E. Marrs, J. R. Henderson, D. A. Knapp, and M. B. Schneider, Phys. Scripta T22, 157 (1988).

[3] P. Beiersdorfer et al., in UV and X-Ray Spectroscopy of Astrophysical and Laboratory Plasmas, ed. by E. Silver and S. Kahn (Cambridge University Press, Cambridge, 1993), p. 59.

[4] P. Beiersdorfer, Nucl. Instrum. Methods B56/57, 1144 (1991).

[5] P. Beiersdorfer, M. H. Chen, R. E. Marrs, M. B. Schneider, and R. S. Walling, Phys. Rev. A 44, 396 (1991).

[6] P. Beiersdorfer, R. E. Marrs, J. R. Henderson, D. A. Knapp, M. A. Levine, D. B. Platt, M. B. Schneider, D. A. Vogel, and K. L. Wong, Rev. Sci. Instrum. 61, 2338 (1990).

[7] P. Beiersdorfer, V. Decaux, S. Elliott, K. Widmann, and K. Wong, Rev. Sci. Instrum., in press.

[8] D. Schneider, D. A. Church, G. Weinberg, J. Steiger, B. Beck, J. McDonald, E. Magee, and D. Knapp, Phys. Rev. A, in press.

[9] D. Schneider, M. A. Briere, J. McDonald, and J. Biersack, Rad. Effects and Defects in Solids 127, 113 (1993). 


\section{Figures}

Fig. 1. Measured line profiles of lines $j$ and $k$ produced by dielectronic recombination of beam electrons with heliumlike $\mathrm{Ti}^{20+}$ ions. The width of each line is determined by the thermal Doppler motion of the emitting ion. (a) high-temperature case where the line widths correspond to a $685-\mathrm{eV}$ ion temperature; (b) low-temperature case where the line widths correspond to a $70-\mathrm{eV}$ ion temperature. 

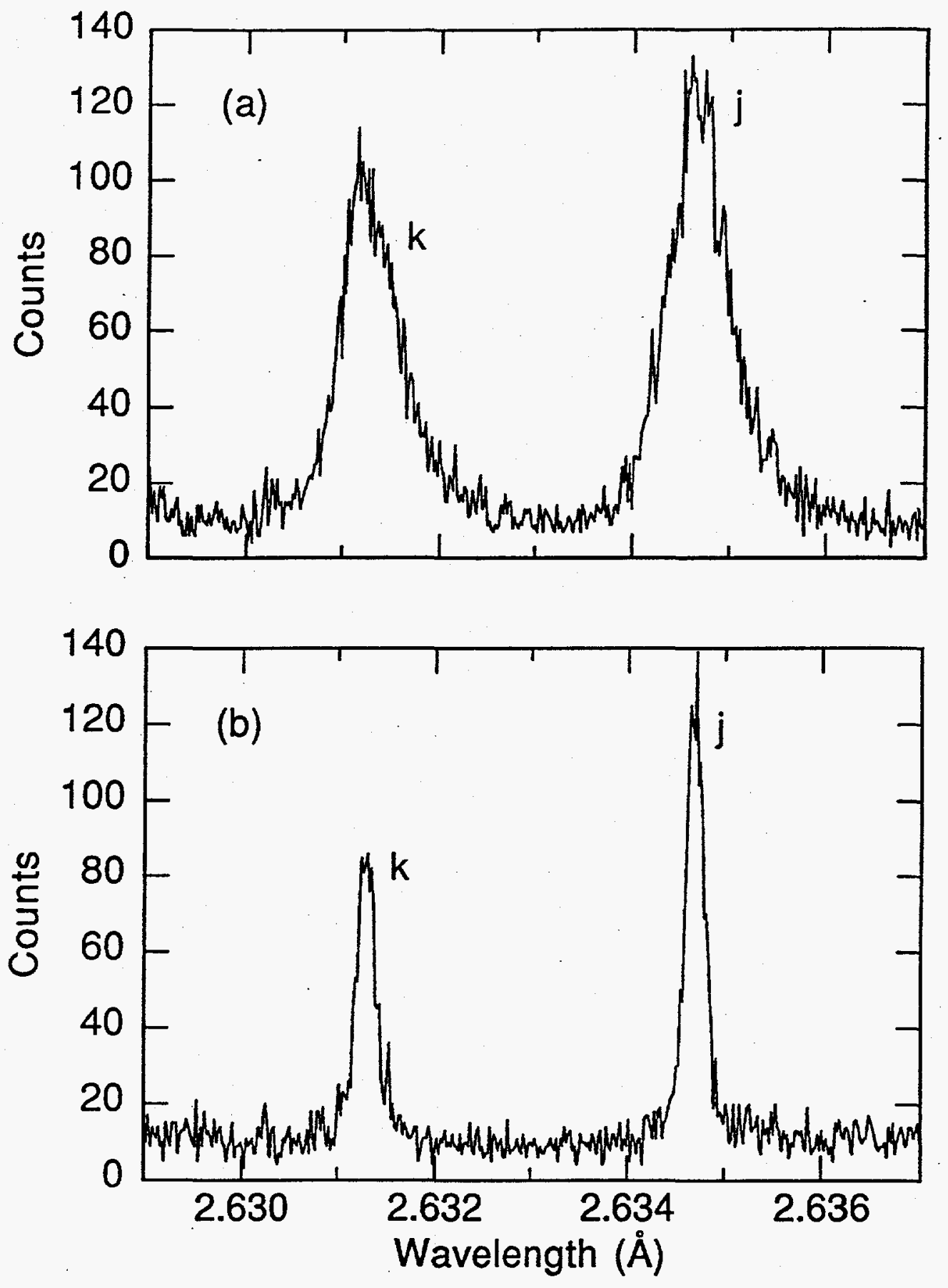

Figure 1. 